\title{
Optical frequency measurement across a 104-THz gap with a femtosecond laser frequency comb
}

\author{
Scott A. Diddams, David J. Jones, Long-Sheng Ma, Steven T. Cundiff, and John L. Hall \\ JILA, University of Colorado and National Institute of Standards and Technology, Boulder, Colorado 80309-0440 \\ Received September 27, 1999 \\ The frequency-domain mode comb of a Ti:sapphire femtosecond laser centered at $350 \mathrm{THz}$ is broadened to \\ $150 \mathrm{THz}$ (full width at $-30 \mathrm{dBc}$ ) by self-phase modulation in a single-mode optical fiber. By phase locking \\ continuous-wave lasers to elements of the comb near 1064 and $778 \mathrm{~nm}$, we measure the 104-THz frequency gap \\ between these two lasers with a relative uncertainty of $2.7 \times 10^{-11}$ in $1 \mathrm{~s}$. (C) 2000 Optical Society of America \\ OCIS codes: $120.3930,320.7090$.
}

Optical frequency standards have the potential benefit over their microwave counterparts of improved relative resolution, owing to an $\sim 10^{4}$ increase in frequency. However, the significantly higher optical frequencies have proved challenging to measure. ${ }^{1}$ Not only is it difficult to multiply up from the $9.193-\mathrm{GHz}$ cesium primary standard but also the coverage of known optical standards is still very sparse, thus inhibiting their use in fundamental spectroscopy and in the characterization of new and potentially improved optical standards. Recently, it was shown that the frequencydomain mode comb associated with a femtosecond ( $\mathrm{fs}$ ) mode-locked laser can be used for precision measurements across frequency gaps of several tens of terahertz. ${ }^{2}$ This novel technique has already affected fundamental atomic physics by permitting a new determination of the fine structure constant $\alpha$ (Ref. 2) and by providing a more direct connection between the cesium microwave standard and the $1 s-2 s$ optical frequency of hydrogen. ${ }^{3}$

Previously we demonstrated the potential to span gaps approaching $100 \mathrm{THz}$ with a 10 -fs laser. ${ }^{4}$ In this Letter we report on our use of $\sim 1.15 \times 10^{6}$ fs laser modes as a frequency scale to measure the $104-\mathrm{THz}$ gap between existing optical standards at 778 and $1064 \mathrm{~nm}$. To our knowledge, this is the largest gap ever bridged with a frequency comb. This measurement also demonstrates the feasibility of phase locking weak elements of the fs comb to a continuous-wave (cw) laser and sets an upper limit on the variation of the fs comb spacing after spectral broadening by self-phase modulation in an optical fiber.

Our measurement is made with the apparatus shown schematically in Fig. 1. Central to the experiment is a Kerr-lens mode-locked Ti:sapphire laser that provides $115 \mathrm{~nm}$ of bandwidth (FWHM) directly from the laser [dashed curve of Fig. 2(a)]. ${ }^{5}$ The laser operates at a $90-\mathrm{MHz}$ repetition rate. The optical spectrum easily covers the 778-nm regime; however, to extend the coverage to $1064 \mathrm{~nm}$ we broaden the frequency comb by self-phase modulation in $2 \mathrm{~m}$ of single-mode silica fiber. ${ }^{6} \quad$ A fused-silica prism pair compensates for the positive dispersion of the output coupler and the other intermediate optics, while additionally providing a small negative frequency chirp so that the pulse compresses to maximum intensity once it enters the fiber. With $120-\mathrm{mW}$ average power coupled into the fiber, the spectrum that emerges, shown in Fig. 2(a), has $\sim 10 \mathrm{pW}$ per mode at $1064 \mathrm{~nm}(-37 \mathrm{dBc})$. This is sufficient power for phase locking.

A small portion of the output pulse train is detected with a fast $\mathrm{p}-\mathrm{i}-\mathrm{n}$ diode, and the 22nd harmonic of the repetition rate is mixed with the $1.979-\mathrm{GHz}$ output of a rf oscillator, thereby providing a phase-dependent error signal for locking the repetition rate. With appropriate amplification and filtering, this error signal is supplied differentially to a two-section tilting piezoelectric transducer mounted behind the flat mirror at the end of the cavity, where the spectrum is spatially dispersed across this mirror by an intracavity prism pair. Tilting of this mirror provides a nearlinear phase change with frequency, which is a group delay for the pulse and therefore controls the repetition rate and the mode spacing. ${ }^{2,3}$ The maximum required tilt angle is $\sim 10^{-4} \mathrm{rad}$, substantially less than the beam divergence, so misalignment of the cavity is negligible. The internal clock of the rf oscillator is referenced to a rubidium microwave standard that has a short-term instability of $2.5 \times 10^{-11}$ for an averaging time of $1 \mathrm{~s}$. The average frequency offset of the rubidium oscillator is measured over one day to the level of a few parts in $10^{13}$ and compared against the National Institute of Standards and Technology ensemble of cesium clocks by common-view global positioning system (GPS) reception. ${ }^{7}$

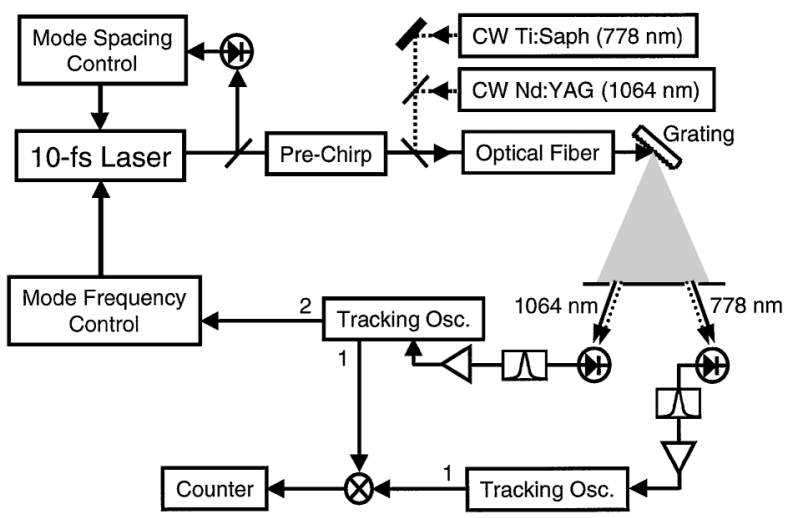

Fig. 1. Block diagram of the apparatus used for measurement of the 104-THz gap between 1064 and $778 \mathrm{~nm}$. Osc's, oscillators. 

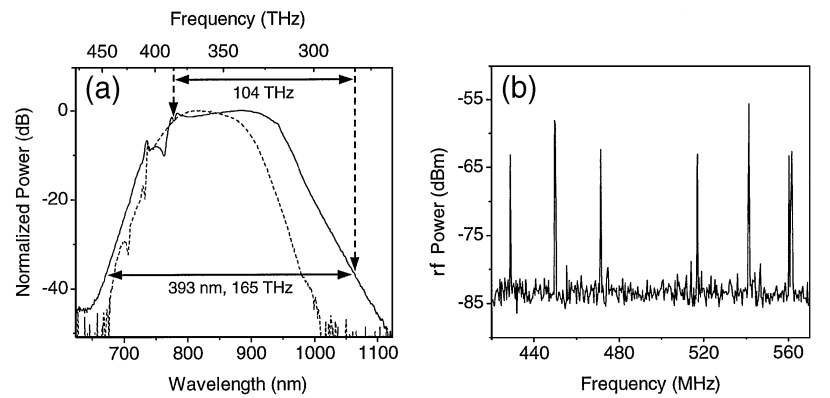

Fig. 2. (a) Optical spectrum directly from the laser (dashed curve) and after broadening in silica fiber (solid curve). The dashed arrows indicate the positions of the cw Ti:sapphire and the Nd:YAG lasers. (b) rf spectrum of the heterodyne signal at $1064 \mathrm{~nm}$ between $\mathrm{cw} \mathrm{Nd:YAG}$ and four elements of the broadened fs comb. Resolution bandwidth, $100 \mathrm{kHz}$; sweep time, $150 \mathrm{~ms}$.

Two stabilized cw lasers are also employed in the measurement. At $778 \mathrm{~nm}$ we use a Ti:sapphire laser that is locked to the $5 S_{1 / 2}(F=3) \rightarrow 5 D_{5 / 2}(F=5)$ two-photon transition in ${ }^{85} \mathrm{Rb}^{8}$ The Comité International des Poids et Mesures (CIPM) recommended value for this transition is $385,285,142,378 \mathrm{kHz} \pm$ $5 \mathrm{kHz}$. At $1064 \mathrm{~nm}$ we use the output of a Nd:YAG laser. The second harmonic of this laser is locked to the $a_{10}$ component of the $R(56) 32-0$ transition in ${ }^{127} \mathrm{I}_{2} \cdot{ }^{9}$ The CIPM-recommended frequency at $532 \mathrm{~nm}$ is $563,260,223,480 \mathrm{kHz} \pm 40 \mathrm{kHz}$. Both cw lasers and the fs output are coupled into the same optical fiber, thus ensuring excellent spatial mode overlap. The output of the fiber is spectrally dispersed by an $1800-$ line $/ \mathrm{mm}$ grating, and heterodyne signals are measured at two $\mathrm{p}-\mathrm{i}-\mathrm{n}$ photodiodes positioned behind slits that pass $\sim 1 \mathrm{~nm}$ of bandwidth at $778 \mathrm{~nm}$ and $1064 \mathrm{~nm}$, respectively.

A second feedback loop on the fs laser controls the optical-frequency position of the entire comb by beating one of its components against $\sim 1 \mathrm{~mW}$ of the stabilized cw Nd:YAG laser and locking this beat at a given offset. The measured rf spectrum (with the locking loop open) of this beat signal is shown in Fig. 2(b). The larger peaks near 450 and $540 \mathrm{MHz}$ are the fifth and sixth harmonics of the laser repetition rate, and the slightly smaller peaks are the heterodyne signals. The position of the heterodyne signals depends on the particular cavity length, and the apparent $45-\mathrm{MHz}$ spacing that can be seen in Fig. 2(b) is coincidental. One of these heterodyne signals is selected with a rf bandpass filter and regenerated with a tracking oscillator, which locks a voltage-controlled oscillator to the relatively noisy beat by use of a balanced mixer as a phase detector. We confirm that cycle slipping does not occur, as the phase difference between the voltagecontrolled oscillator and the tracked beat is kept less than $\pi / 6 \mathrm{rad}$. A filtered version of the balanced mixer output from the tracking oscillator (port 2 in Fig. 1) is also applied to a standard piston-mode piezoelectric transducer mounted behind the output coupler of the laser. Such a transducer will normally change both the frequency position and the spacing of the comb. However, we can electrically orthogonalize the control of these two variables by adding part of this piston input to the tilting piezoelectric transducer described above. Thus, to first order, this piston-mode input varies only the optical frequency position of the entire comb.

When the feedback loops controlling the mode position and the mode spacing are locked, a second tracking oscillator is used to regenerate the heterodyne signal between the $\sim 0.5 \mathrm{~mW}$ of $\mathrm{cw}$ Ti:sapphire and the fs comb. In this spectral regime the signal-to-noise ratio of the tracked beat is typically $10-20 \mathrm{~dB}$ greater than what is obtained at $1064 \mathrm{~nm}$. The regenerated beats at both 778 and $1064 \mathrm{~nm}$ (ports 1 in Fig. 1) are subsequently mixed, and the sum or difference frequency is counted and recorded. As the sign of the frequency differences between the two cw lasers and their respective fs comb lines is ambiguous, knowing only the rf spectrum, we must consider four possibilities in the determination of the 104-THz gap. For this purpose it is convenient to express the measured frequency of the Nd:YAG radiation $\left(f_{1064}\right)$ in terms of the 778 -nm frequency $\left(f_{778}\right)$ as

$$
f_{1064}=f_{778}-n \Delta \pm(778 \mathrm{~nm} \text { beat } \pm 1064 \mathrm{~nm} \text { beat })
$$

where $n$ is an integer and $\Delta$ is the mode spacing of the fs laser as determined from the rf oscillator that controls the repetition rate. With the mode spacing of the fs comb fixed, the variations of the two beats at 1064 and $778 \mathrm{~nm}$ are correlated as the comb position shifts. Depending on the frequencies of the two cw lasers relative to their respective fs comb lines, this correlated noise is removed by measurement of either the difference or the sum of the two beats. In this way we can eliminate two of the possible solutions to Eq. (1). Because both $f_{1064}$ and $f_{778}$ are already known to much better than $1 \mathrm{MHz}$, we remove the remaining ambiguous sign of Eq. (1) by incrementing or decrementing $n$. Although it is demonstrated here for two previously measured standards, this technique is nonetheless quite general, as a common laboratory wavemeter has sufficient resolution with which to determine an unknown frequency to $<10 \mathrm{MHz}$, well below one half of the $90-\mathrm{MHz}$ mode spacing of the fs laser.

The results of measurements taken over a 23-day period are shown in Fig. 3, in which we compare our determination of $f_{1064}$ with the CIPM-accepted value. Each plotted point is the computed mean of a data set of 25 or 50 counter readings (recorded with a 1-s gate time), and the error bars are the standard deviation of the readings in each data set. The significant decrease in the error bars after two days is the result of an improved signal-to-noise ratio in the rubidium twophoton lock. The average of the 43 mean values yields an offset from the accepted frequency of $+23.2 \mathrm{kHz}$, whereas the standard deviation of the means is $930 \mathrm{~Hz}$. The inset of Fig. 3 is a histogram of $\sim 800$ counter readings taken on and after the third day. A Gaussian fit to this distribution yields a mean of $23.1 \mathrm{kHz}$ with a standard deviation of $2.8 \mathrm{kHz}$, which represents a fractional uncertainty of $\sim 2.7 \times 10^{-11}$ in $1 \mathrm{~s}$ for the 


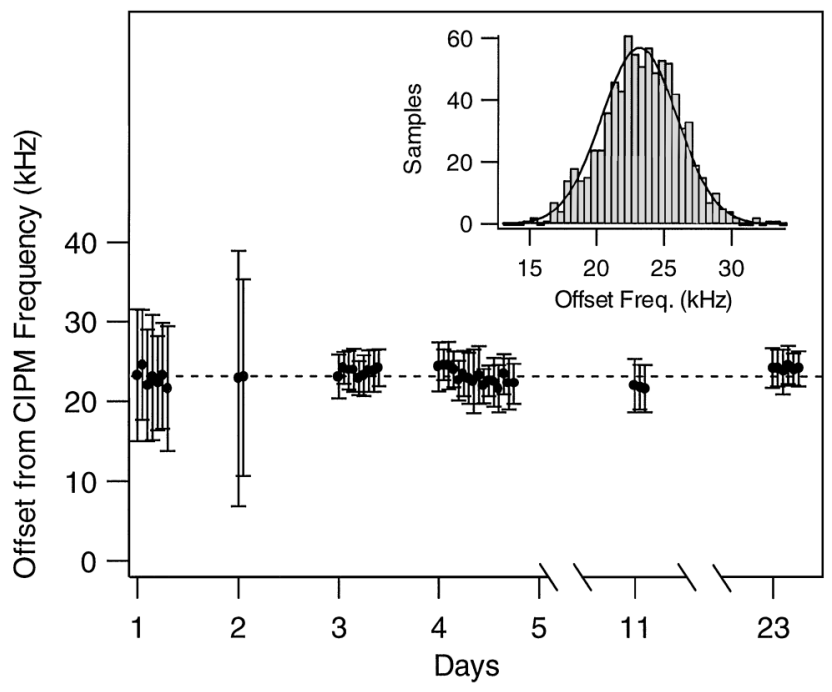

Fig. 3. Summary of measured Nd:YAG frequency $f_{1064}$ with respect to the CIPM recommendation of $281,630,111$, $740 \mathrm{kHz}$. The dashed line is the average of all the plotted points $(23.2 \mathrm{kHz})$. The inset is a histogram of the $\sim 800$ individual measurements on and after the third day.

$104-\mathrm{THz}$ gap. This variation is near the expected limits set by the rubidium microwave oscillator that controls the mode spacing and the rubidium twophoton spectrometer that guides the cw Ti:sapphire laser. It is promising that the stability and day-today reproducibility of these initial measurements do not appear to be fundamentally limited by the fs laser or the measurement concept.

The presence of the $23.1-\mathrm{kHz}$ offset in the results of Fig. 3 should not yet lead to the conclusion that the CIPM value of $f_{1064}$ requires correcting, as all systematic errors have not been characterized. In particular, the accuracy of our rubidium two-photon spectrometer has not been confirmed against a known standard. With this relatively untested technique, there could also be unknown offsets that result from the nonlinearities involved in the generation of the extremely broad spectrum. Udem et $a l .^{2}$ have shown that the mode spacing directly from the laser is constant to 3 parts in $10^{17}$ over a $20-\mathrm{THz}$ bandwidth ${ }^{2}$; however, this has not been verified with the fiber-broadened spectrum of Fig. 2. To set an upper limit on variations of the mode spacing in our situation we have compared the repetition rate of the laser at 1064 and $778 \mathrm{~nm}$. The $90-\mathrm{MHz}$ pulse train is detected at both wavelengths, and the two signals are mixed in quadrature. The output of the mixer, representing the phase difference between the two $90-\mathrm{MHz}$ signals, is observed to be nearly constant at the 20 -mrad level over thousands of seconds. In the worst case, phase variations on a 10-s time scale indicate that the repetition rate in the two spectral regions differs by no more than $0.2 \mathrm{mHz}$. This, however, should be considered a generous upper limit, for the detected phase difference was clearly sensitive to thermal fluctuations. If the mode spacing were assumed to vary linearly, this $0.2-\mathrm{mHz}$ difference would accumulate to a frequency offset of a few hundred hertz across the 104-THz gap.

Looking to the near future, the rapid trend in new ultrabroadband sources ${ }^{11}$ and spectral-broadening optical fibers ${ }^{12}$ suggests that it will be possible to use the fs comb to bridge an entire optical octave. Furthermore, we note that our frequency-domain control of the fs mode comb provides time-domain pulses with a controllable offset between the pulse envelope and the carrier wave. ${ }^{2,3,12}$ Such unprecedented control of the phases of optical fields on a sub-fs time scale will likely open a new realm of nonlinear optics with pulses consisting of just a few cycles.

The authors are most grateful to T. Hänsch and his group for sharing their results before publication. We appreciate the assistance of Jun Ye and Judah Levine. Funding was provided by the National Science Foundation and the National Institute of Standards and Technology. S. A. Diddams's e-mail address is sdiddams@jila.colorado.edu.

\section{References}

1. D. A. Jennings, K. M. Evenson, and D. J. E. Knight, Proc. IEEE 74, 168 (1986).

2. Th. Udem, J. Reichert, R. Holzwarth, and T. W. Hänsch, Phys. Rev. Lett. 82, 3568 (1999); Opt. Lett. 24, 881 (1999).

3. J. Reichert, R. Holzwarth, Th. Udem, and T. W. Hänsch, "Managing the frequency of light with modelocked lasers," Opt. Commun. (to be published).

4. J. L. Hall, M. S. Taubman, S. A. Diddams, B. Tiemann, J. Ye, L.-S. Ma, D. Jones, and S. T. Cundiff, "Stabilizing and measuring optical frequencies," in Proceedings of the International Conference on Laser Spectroscopy (World Scientific, to be published).

5. M. T. Asaki, C.-P. Huang, D. Garvey, J. Zhou, H. C. Kapteyn, and M. M. Murnane, Opt. Lett. 18, 977 (1993).

6. K. Imai, M. Kourogi, and M. Ohtsu, IEEE J. Quantum Electron. 34, 54 (1998).

7. J. Levine, in Proceedings of 1998 IEEE International Frequency Control Symposium (Institute of Electrical and Electronics Engineers, New York, 1998), pp. 284-291.

8. D. Touahri, O. Acef, A. Clairon, J.-J. Zondy, R. Felder, L. Hilico, B. de Beauvoir, F. Biraben, and F. Nez, Opt. Commun. 133, 471 (1997).

9. J. L. Hall, L.-S. Ma, M. Taubman, B. Tiemann, F.-L. Hong, O. Pfister, and J. Ye, IEEE Trans. Instrum. Meas. 48, 583 (1999).

10. U. Morgner, F. X. Kärtner, S. H. Cho, Y. Chen, H. A. Haus, J. G. Fujimoto, E. P. Ippen, V. Scheuer, G. Angelow, and T. Tschudi, Opt. Lett. 24, 411 (1999); D. H. Sutter, G. Steinmeyer, L. Gallmann, N. Matuschek, F. Morier-Genoud, U. Keller, V. Scheuer, G. Angelow, and T. Tschudi, Opt. Lett. 24, 631 (1999).

11. J. Ranka, R. Windeler, and A. Stentz, Opt. Lett. 25, 25 (2000).

12. H. R. Telle, G. Steinmeyer, A. E. Dunlop, J. Stenger, D. H. Sutter, and U. Keller, Appl. Phys. B 69, 327 (1999). 